\title{
Study of 144-Channel Multi-Anode Hybrid Avalanche Photo-Detector for the Belle II RICH Counter
}

\author{
I. Adachi ${ }^{* a}$, R Dolenec ${ }^{b}$, K. Hara $^{a}$, M. Higuchi ${ }^{c}$, T. Iijima $^{d}$, S. Iwata ${ }^{e}$, H. Kakuno ${ }^{e}$, \\ H. Kawai ${ }^{f}$, T. Kawasaki ${ }^{g}$, S. Korpar ${ }^{b, h}$, P. Križan ${ }^{b, i}$, T. Kumitaa ${ }^{e}$ S. Nishida ${ }^{a}$, W. Mori ${ }^{j}$, \\ S. Ogawa ${ }^{j}$, R. Pestotnik ${ }^{b}$, Y. Sakashita ${ }^{e}$, L. Santelj ${ }^{b}$, A. Seljak ${ }^{b}$, T. Sumiyoshi ${ }^{e}$, \\ H. Takagaki ${ }^{e}$, M. Tabata ${ }^{f, k}$, Y. Yusa ${ }^{g}$, R. Verheyden ${ }^{b}$ \\ a) IPNS, High Energy Accelerator Research Organization (KEK), Tsukuba, Japan \\ b) Jožef Stefan Institute, Ljubljana, Slovenia \\ c) Tokyo University of Science, Noda, Japan \\ d) Nagoya University, Nagoya, Japan \\ e) Tokyo Metropolitan University, Hachioji, Japan \\ f) Chiba University, Chiba, Japan \\ g) Niigata University, Niigata, Japan \\ h) University of Maribor, Maribor, Slovenia \\ i) University of Ljubljana, Ljubljana, Slovenia \\ j) Toho University, Funabashi, Japan \\ k) Japan Aerospace Exploration Agency (JAXA), Sagamihara, Japan \\ E-mail: ichiro.adachi@kek.ip
}

\begin{abstract}
We have developed a new hybrid avalanche photo-detector (HAPD) as a photon sensor for the Belle II RICH counter. We investigated the basic device characterizations including single-photon response. In addition, radiation damage due to $\gamma$ rays and neutrons was studied in detail. Furthermore, we report on the results of a test beam experiment carried out using a prototype consisting of 6 photo-detectors. We successfully observed a clear Cherenkov image and the $\pi / K$ separation power was obtained to be more than $5 \sigma$ at $4 \mathrm{GeV} / \mathrm{c}$.
\end{abstract}

International Workshop on New Photon-detectors,

June 13-15, 2012

LAL Orsay, France

* Speaker. 


\section{Introduction}

The second generation of high-luminosity $e^{+} e^{-}$collider project, the SuperKEKB/Belle II, has been approved. Particle identification, in particular the identification of $\pi$ and $K$ particles, has been the essential role in various physics analyses carried out at Belle. Our detector upgrade that is currently underway aims at strengthening $\pi / K$ separation capability with higher background environment predicted at Belle II. The present threhold-type aerogel Cherenkov counter located at the end-cap is to be replaced by a proximity focusing ring imaging Cherenkov (RICH) counter based on a silica aerogel radiator. The current end-cap geometry acutely limits the space available and a proximity focusing with an expansion distance of $200 \mathrm{~mm}$ is a unique choice. The photon sensor is one of the most essential elements in the RICH counter. Our requirements as regards this sensor are the following: (1) high sensitivity to a single photon, (2) position resolution of $\sim 5 \times 5 \mathrm{~mm}^{2}$, (3) large detection area, and (4) immunity to an axial magnetic field. To satisfy these requirements, we have been developing a new hybrid avalanche photo-detector (HAPD) as a dedicated photon sensor in collaboration with Hamamatsu Photonics K.K. In this paper, performance studies done with HAPD samples are presented. Fundamental feature of HAPDs, including a test in the presence of a magnetic field, is described in section 2, and section 3 reports on the various tests regarding radiation damage. The beam test results are mentioned in section 5 and section 6 concludes this paper.

\section{HAPD fundamental performance}

\begin{tabular}{|c|c|}
\hline Package size & $73 \times 73 \mathrm{~mm}^{2}$ \\
Package height & $30 \mathrm{~mm}$ \\
Effective area & $64 \%$ \\
$\#$ of pixels & $12 \times 12(6 \times 6 /$ APD chip $)$ \\
Pixel size & $4.9 \times 4.9 \mathrm{~mm}^{2}$ \\
Total gain & $\sim 10^{5}$ \\
Input capacitance & $80 \mathrm{pF}$ \\
\hline
\end{tabular}

Table 1: Summary of the HAPD specifications.

The HAPD consists of a vacuum tube coupled with four avalanche photo-diodes (APDs), each of which is pixelated into $6 \times 6$ pads, and the area of each pad is $4.9 \times 4.9 \mathrm{~mm}^{2}$. A 3 -mm-thick quartz window, on which a bi-alkali photocathode is instrumented, is attached to a ceramic tube housing four APDs, resulting in total number of pixels of 144. Photoelectrons generated at the photocathode are accelerated via an electric field produced by a high voltage of $-7 \sim 8 \mathrm{kV}$, and they are bombarded onto the APD. Table 1 summarizes our HAPD specifications. The typical peak quantum efficiency $(\mathrm{QE})$ of the photocathode is about $27 \%$, and a recent study shows that a $\mathrm{QE}$ of more than $30 \%$ can be achieved [U].

The pulse height spectrum was examined by illuminating LED light. The obtained distribution is shown in Figure 田, where a clear single-photon peak can be observed. From this plot, the signal-to-noise $(\mathrm{S} / \mathrm{N})$ ratio for single-photon signal separation defined as $G / \sigma_{\text {ped }}$, where $G$ and $\sigma_{p e d}$ represent the total gain and the pedestal peak sigma, respectively, was extracted to be $\sim 26$. 


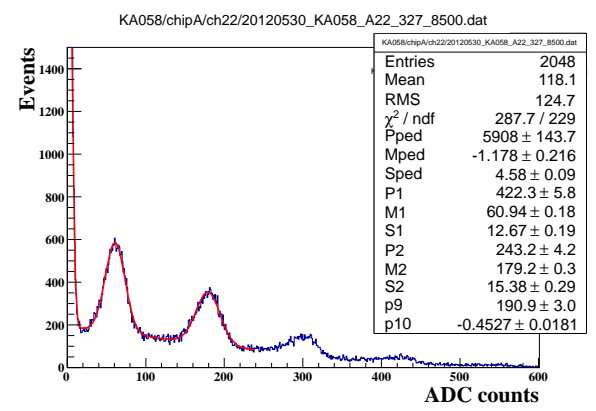

Figure 1: Pulse height distribution.
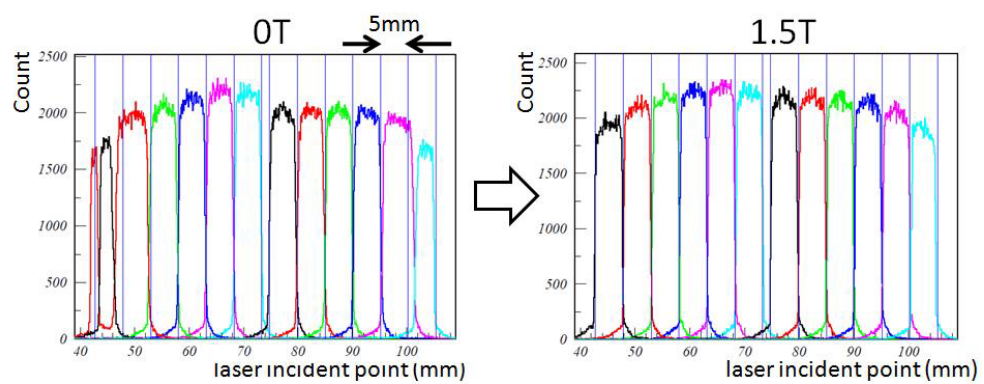

Figure 2: Hit distribution for one row in HAPD with (right) and without (left) application of magnetic field of 1.5 tesla.

The single-photon sensitivity for the entire photocathode surface was measured via two-dimensional scanning with a light source. Signals from all the pads of the HAPD were processed with a custommade ASIC readout system, where the amplification and the digitization of input signal were done []. The light intensity was adjusted to be equivalent to that of a single photon. The hit distribution obtained by scanning one row in the HAPD is indicated in Figure $\square$ (left). The same measurement was carried out under an axial magnetic field of 1.5 tesla as shown in Figure $\square$ (right). Good responses were observed both with and without a magnetic field.

\section{Radiation damage}

The results from recent studies have predicted serious beam-related background issues in our photon detector environment in the Belle II structure [3]. Our target is to ensure that HAPDs are radiation-resistant up to values of $1000 \mathrm{~Gy}$ for $\gamma$ rays and up to $10^{12} / \mathrm{cm}^{2}$ for neutrons, where neutron flux is calculated using $1 \mathrm{MeV}$ equivalent energy. Several intensive studies have been carried out with this aim.

\subsection{Ionization damage}

A series of $\gamma$-ray irradiation tests was carried out using the ${ }^{60} \mathrm{Co}$ irradiation facility at Nagoya University, and various types of APDs were tested. In the first test, a quick increase in leakage current was observed. Based on detailed studies, this phenomenon mainly comes from the surface film (alkali shielding film) that was introduced to prevent alkali ions during the photocathode fabrication from contacting to the APD surface. The incident of $\gamma$ rays leads to local charge accumulation 


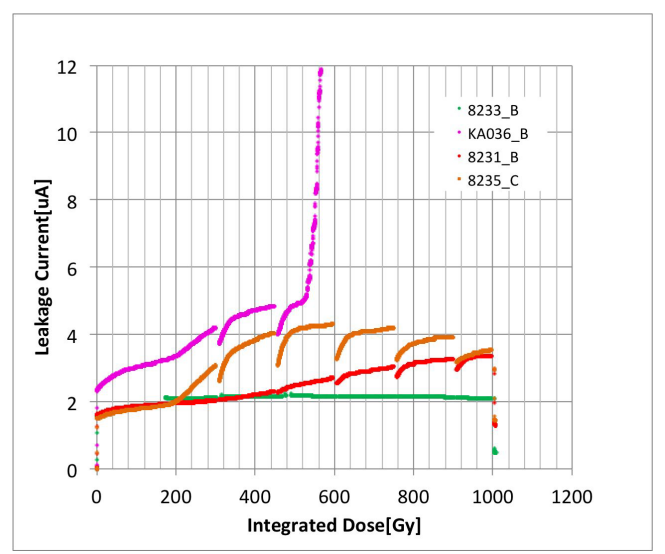

Figure 3: Leakage current $(\mu \mathrm{A})$ as a function of integrated dose(Gy) for various APD types.

around this film, and this generates a potential difference in this area. Once this difference exceeds a certain threshold, electric breakdown occurs, thereby causing rapid rise in leakage current. To stop the occurrence of this phenomenon, the alkali shielding film was modified to mitigate charge accumulation. Figure [3 shows the measured leakage currents as a function of $\gamma$-ray irradiation doses up to $1000 \mathrm{~Gy}$ for the four different types of APDs. As observed from this figure, the KA36 APD type shows a rapid increase in leakage current when the integrated dose exceeds 520 Gy, whereas the other types show small or almost no increase even at $1000 \mathrm{~Gy}$. From these results, the soon-to-be fabricated HAPD will use radiation-tolerant APDs.

\subsection{Neutron radiation damage}

Exposure to neutron dose is another important concern since bulk damage of solid state detectors due to neutron irradiation has been reported in other experiments [困]. A large leakage current in the APD corresponds to a significant amount of noise, thereby leading to the deterioration of the $\mathrm{S} / \mathrm{N}$ ratio of the single-photon signal. We performed irradiation tests several times at nuclear reactor facility "Yayoi" at University of Tokyo [5]. APD samples were exposed to a neutron integrated dose of $4 \times 10^{12} / \mathrm{cm}^{2}$. From this study, we found that reducing the thickness of the layer responsible for the avalanche process along with the use of flexible readout electronics having shorter shaping times was essential to improve the $\mathrm{S} / \mathrm{N}$ ratio for a single photon. Details of these investigations can be found in [可. The most recent irradiation test was carried out in this June at the J-PARC MLF BL10 beam facility [ $\left[\right.$ ] . In this test, we irradiated the modified HAPD samples up to $10^{12}$ $/ \mathrm{cm}^{2}$ and we measured the leakage current and pulse height for the single-photon signal. Figure 6 is a single-photon spectrum of the APD samples 30 days after exposure to neutron irradiation. As observed in this figure, sufficient single-photon sensitivity is still retained after $10^{12} / \mathrm{cm}^{2}$ neutron irradiation.

\section{Test beam experiment}

We performed a test beam experiment at the CERN SPS facility in September 2011 using a prototype counter. In this prototype, six HAPDs were arranged in the same manner as that to be 


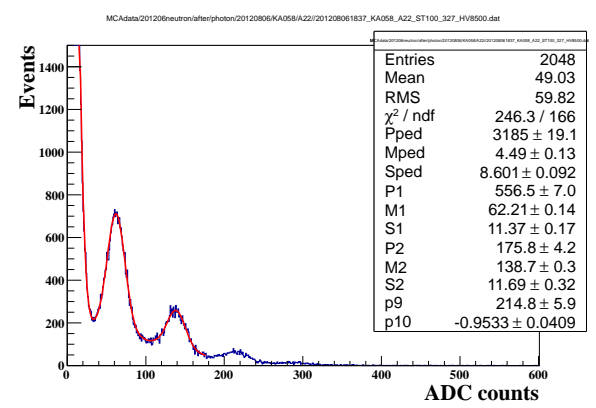

Figure 4: Single-photon signal 30 days after neutron irradiation test.

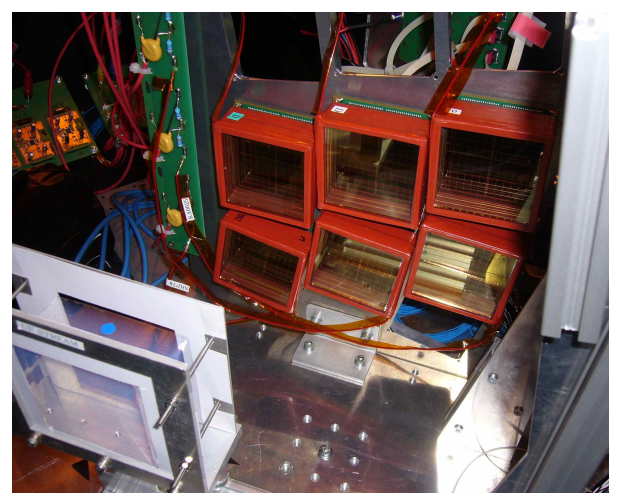

Figure 5: Photograph of prototype counter.

deployed in the real detector geometry. The readout electronics system was basically identical to that used in two dimensional scan described in section 2. Our prototype is shown in Figure 5 . The track parameters were calculated from the information obtained using two MWPCs located upstream and downstream in the prototype. Two layers of aerogel tiles, each of $20 \mathrm{~mm}$ thickness, were positioned as Cherenkov radiators [ $[\mathbb{8}]$. The refractive index combination was selected as 1.045 and 1.055 for the upstream and downstream radiators, respectively, so that the Cherenkov photons generated from the two tiles formed an overlapped ring image onto the HAPD surface. Figure (top) shows the obtained ring image for this radiator setup, and the bottom figure shows the Cherenkov angle distribution. A clean peak of the Cherenkov angle was detected. By fitting this distribution with a Gaussian function, the Cherenkov angle resolution for a single photon was obtained to be $\sigma_{\text {single }}=14.1 \mathrm{mrad}$. The number of photoelectrons was extracted by integrating the fitted function over $\pm 3 \sigma_{\text {single }}$, thereby yielding $N_{p e}=11.4$. From these results, the Cherenkov angle resolution defined as $\sigma=\sigma_{\text {single }} / \sqrt{N_{p e}}$ was obtained to be $4.17 \mathrm{mrad}$. Consequently this result demonstrates $5.5 \sigma \pi / K$ separation capability at $4 \mathrm{GeV} / \mathrm{c}$.

\section{Conclusions}

We have been conducting development of new HAPD for the Belle II RICH counter, and we obtained an excellent single-photon response from the currently developed HAPD samples. Furthermore, we studied the effect of radiation damage due to $\gamma$ rays and neutrons, and consequently 

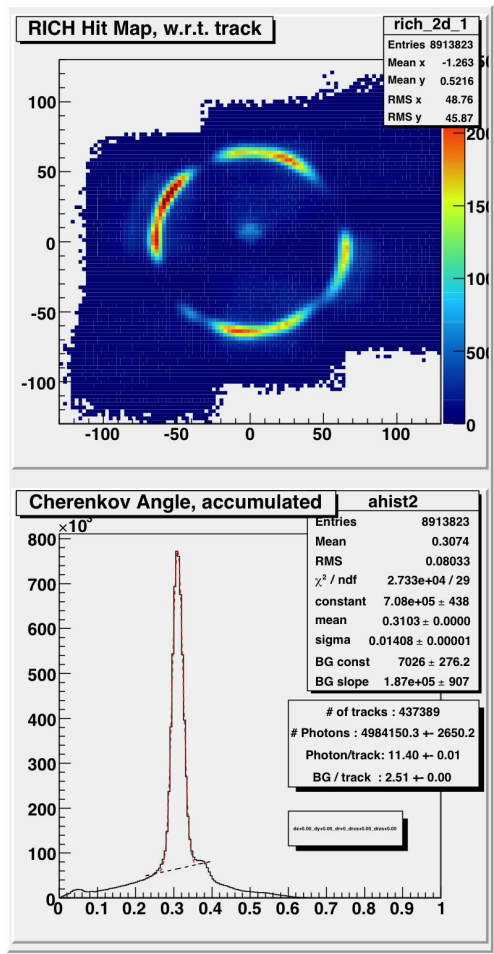

Figure 6: top: Cherenkov ring image. bottom: Cherenkov angel distribution.

we have ensured improved radiation tolerance by modifying the APD structure. We carried out a beam test study at CERN using a prototype counter and a separation capability of more than $5 \sigma$ $\pi / K$ was obtained. We plan to move to the HAPD fabrication phase from the R\&D phase in the near future.

\section{Acknowledgements}

Authors would like to thank Belle II colleagues for their supports. This work was partially supported by JSPS KAKENHI Grant Number 21105005.

\section{References}

[1] Hamamatsu Photonics K.K., private communication.

[2] A. Seljak et al., JINST 6(2011) C12051.

[3] M. Iwasaki et al., Conf. Proc. C1205201(2012)1825.

[4] M. Moll et al., Nucl. Instr. Meth. A426(1999)87.

[5] Yayoi reactor, http://www.tokai.t.u-tokyo.ac.jp/ rokan/index.html.

[6] S .Iwata et al., contributed to TIPP2010, Chicago, 2010.

[7] J-PARC MLF, http://j-parc.jp/MatLife/en/index.html.

[8] I. Adachi et al., Nucl. Instr. Meth. A639(2011)222. 